Aim of the study: Evaluation of FLT/PET/ CT usefulness in diagnosis and qualification for surgical treatment of gastric cancer.

Material and methods: The FLT/PET/CT test was carried out in a group of 50 gas tric cancer patients. Based on the test result, a decision followed about the therapeutic procedure to be applied. A comparison was made with regards to the consistency of the cancer growth advancement degree evaluation in the initial preoperative FLT/PET/CT test against the evaluation of postoperative degree of cancer advancement in histopathology. Results: In the group of 50 diagnosed patients a surgical treatment was used for 37 patients. 21 resections were performed out of which 19 operations were radical In the group of 16 non-resective operations 2 post-laparotomic patients were selected for inductive treatment. In the group of 13 patients who did not undergo any surgery, 10 were directed to palliative care and 3 for inductive treatment. In the group of 50 patients, the applied FLT-PET/CT test confirmed presence of primary tumor in 49 patients. The presence of increased uptake of FLT in the local lymph nodes during the preoperative FLT-PET/CT test was confirmed in 22 cases. In 14 patients with FLT-PET/Ct N(+) with the M(-) feature resection surgery was performed. The increased uptake of FLT in localizing metastases (nodal and non-nodal) FLT-PET/CT $(\mathrm{M}+)$ was detected in 22 patients. The pres ence of nodal metastases in the postoperative histopathology examination ( $\mathrm{hpN}+$ ) was detected in 14 cases. In these cases preoperative FLT-PET/CT test proved the $\mathrm{N}(+)$ feature in 11 patients. The result FLT-PET/CT N $(-)$ was truly negative in $2 \mathrm{pa}-$ tients, and false negative in 1 patient. In the group of 7 operated hpN(-) patients, in 3 patients a preoperative result FLT-PET/ $\mathrm{CT} \mathrm{N}(+)$ (false positive result) was obtained. The consistency (positive) of nodal metastases identification in FLT-PET/CT as compared to post-surgical histopathology examination scored $11 / 15$, which equals $73.3 \%$. In the group of patients in whom resection surgery was performed, 4 false negative results were obtained $[\mathrm{hp}(\mathrm{N}+)$, FLT-PET/CT (N-)] and 3 false positive results [hp(N-), FLT-PET/CT N(+)].

Conclusions: The initial test results indicate that FLT-PET/CT is an effective method in evaluating the primary tumor and the regional lymph nodes and is useful and beneficial in the diagnosis and further treatment evaluation of gastric cancer. FLT-PET/CT examination facilitates making proper therapeutic decisions it allows the number of unnecessary laparotomies to be lowered.

Key words: FLT PET/CT, gastric cancer diagnosis, histopathology staging, comparison.

\section{Evaluation of FLT-PET/CT usefulness in diagnosis and qualification for surgical treatment of gastric cancer}

\author{
Tomasz Staniuk, Wojciech Zegarski, Bogdan Małkowski, Michał Jankowski, \\ Michat Klag, Tomasz Pietrzak
}

Oncology Centre - Prof. Lukaszczyk Memorial Hospital, Bydgoszcz, Poland

\section{Introduction}

Stomach cancer is one of the most prevalent primary digestive tract malign cancers amongst adults. The still most common radical treatment method remains stomach resection along with the local lymph glands. Preoperative inductive chemotherapy also proves useful in cases of substantial locoregional growth, or radio-chemotherapy (as an elementary treatment method) in the case of higher growth degrees [1-3].

The prognosis depends on the degree of histopathological grading of the cancer, the depth and the diameter of the original tumor's infiltration, the presence of lymph node metastases as well as remote metastases (degree of clinical tumor advance). Over $95 \%$ of the patients treated at the early cancer stages, with cancer limited to the mucous and submucous layer of the gastric wall, survive the period of 10 years [4]. Around $60 \%$ of patients with TNM stage II gastric cancer survive up to 5 years. In stage III, the 5-year survival rate is limited to $30 \%$. But with the cancer spread to remote body areas the 5 -year survival period is achieved by only $5 \%$ of patients [5]. In 2010 research was published reporting the data of a 15-year observation of patients treated with resection of stomach cancer. The survival period of the patients who underwent resection combined with D2 lymphadenectomy increased to 29\%.

The basic diagnostic test when stomach cancer is suspected remains endoscopy of the upper part of the digestive tract, along with tissue sampling of the primary tumor. Evaluation of the stomach cancer advancement is based on imagining tests - the most common tests remain ultrasonography of the abdominal area, radiographic scan of the chest and computed tomography of the abdomen and chest. These tests allow a visual presentation of the primary tumor, of the metastatic lymph nodes as well as an image of remote metastases. However, such tests do not differentiate sufficiently between cancer and inflammatory changes. At the same time, in such tests it is difficult to identify a cancerous process in lymph nodes, when it is not accompanied by a parallel size increase of the lymph glands in the imagining tests [7]. Similar diagnostic difficulties occur when suspicious lesions of metastatic nature are detected, e.g. tumors in the liver, lungs or adrenal glands. A modality which grants a more apt and conclusive diagnosis of cancerous lesions is PET/CT a hybrid combination of positron emission tomography and computed tomography.

Positron emission tomography has been for many years used in diagnostics and monitoring of cancer lesions [8]. Nowadays PET/CT is used in diagnostics of cancer of the esophagus, lungs, breast, uterus, pancreas, large bowel, melanoma, as well as in searching for the site of the primary tumor in metastatic lesions found in the lungs. The most commonly used radiomarker in PET/CT is ${ }^{18} \mathrm{~F}$-fluorodeoxyglucose (FDG), which uses the glucose metabolic path The increased metabolism of FDG is characteristic of many cancer 
types. Not only FDG but also other radiomarkers prove useful in various cancer types [9]. Below, a number of metabolic pathways together with their radiomarkers are mentioned, important for oncology and detectable through PET [10]:

- ${ }^{11} \mathrm{C}$-thymidine, ${ }^{18} \mathrm{~F}$-thymidine (FLT) - replication of DNA, cellular proliferation,

- ${ }^{11} \mathrm{C}$-methionine (MET), ${ }^{18} \mathrm{~F}$-ethyl-tyrosine (FET), ${ }^{18} \mathrm{~F}$-methyltyrosine (FMT), ${ }^{18} \mathrm{~F}$-dihydroxy-phenylalanine (F-DOPA) - protein synthesis, amino acid transport,

- ${ }^{18} \mathrm{~F}$-acetate, ${ }^{11} \mathrm{C}$-choline, ${ }^{18} \mathrm{~F}$-choline $(\mathrm{FCH})$ - membrane lipid synthesis,

- ${ }^{18} \mathrm{~F}$-misonidazole (FMISO), 64 CU-copper-ATSM - hypoxia,

- ${ }^{18} \mathrm{~F}$-annexin $\mathrm{V}$ - apoptosis,

- ${ }^{18}$ F-galacto-RGD - angiogenesis,

- ${ }^{18}$ F-deoxy-arabinofuranosyl-nucleosides (FEAU, FIAU, FMAU) - reporter genes,

- ${ }^{18}$ F-uracil (FU) - tumor therapy control,

- ${ }^{18}$ F-oestradiol (FES) - receptor binding (estrogen),

- ${ }^{68} \mathrm{Ga}$-DOTATOC/DOTANOC - receptor binding (somatostatin).

With gastric cancer, having a variety of histopathological types (carcinoma tubulare, papillare, mucosinum, mucocellulare, planoepitheliale, adenoplanoepitheliale, microcellulare, male at non-differentium), so far the attempts at using the PET/CT test along with fluorodeoxyglucose have not brought the expected results. On account of a low level of glucose metabolism in mucinosum/mucocellulare cancer types (these cancer types have a low level of cellularity and a low number of glut-1 receptors on the cancer cell surface), such cancer types have often not been identified in the FDG$\mathrm{PET} / \mathrm{CT}$ test [11].

In our FDG-PET/CT pilot test of 16 patients, 30\% of stomach cancers were not evidenced through imaging [12]. Similar results have been obtained by other authors [13-19]. Therefore trials have been performed using different radiomarkers in stomach cancer diagnostics. One such marker is ${ }^{18} \mathrm{~F}$-fluorothymidine - an analogue of thymidine appropriated in the metabolic route of cellular divisions. In 2007 Hermann, Ott and Buck published pioneering research, in which the use of ${ }^{18} \mathrm{~F}$-fluorothymidine in the PET test in 45 stomach cancer patients was evaluated. The use of FLT was characterized by high, $100 \%$ sensitivity of primary tumor detection (through FLT-PET), as compared to 69\% sensitivity of a test using ${ }^{18} \mathrm{~F}$-fluorodeoxyglucose (FDG-PET/CT) [20]. The main objective of our prospective research was to assess the value of ${ }^{18} \mathrm{~F}$-fluorothymidine use in PET/CT in stomach cancer diagnostics as well as to define how the results we obtained could be used in qualifying for surgical treatment.

\section{Material and methods}

The group under scrutiny consisted of 50 patients (29 women and $21 \mathrm{men}$ ), with histopathologically confirmed primary stomach cancer. Mean age was 62.5 years (29-84). Between February 2010 and February 2011 all the patients underwent the FLT-PET/CT test at the Nuclear Medicine Department of Oncology Centre - Prof. Lukaszczyk Memorial Hospital in Bydgoszcz. The mean age of all the patients averaged 62.5 (ranging between 29 and 85 years of age). The diagnostic method involved gastroscopy along with tissue sampling, ultrasonography and computed tomography of the whole abdomen area. Once the histopathology result confirmed the presence of stomach cancer, the FLT-PET/CT test was performed. The results of all these tests collectively were used to define the cancer clinical advancement degree. Based on their assessments the patients were assigned to subsequent treatment modes: inductive chemotherapy (2 patients), inductive brachytherapy (1 patient), surgery (37 patients) and palliative chemotherapy (10 patients). In 21 out of 37 patients who underwent the surgery, resection was performed. Tissue samplings were scrutinized in detailed histopathology. The histopathology tests were compared with FLT-PET/CT test results in reference to the primary tumor and metastatic lymph nodes. The degree of tumor identification was evaluated in relation to its histopathological type and its size; also the number of lymph nodes as well as the number of metastatic lymph nodes were counted.

To carry out this study, approval was obtained from the Ethical Committee at Ludwig Rydygier's Bydgoszcz Collegium Medicum Medical University in Torun.

\section{Interpretation of $\mathrm{PET/CT}$ result}

Computed imaging obtained through the PET test reveals quantitative events. The test result (through a color on the scan) conveys the real activity (the degree of intensity of metabolism) of the administered marker in the examined body organ.

In clinical practice, in result descriptions a gradient is used called a standardized uptake value (SUV). This value gradient is defined as a relation between the marker's activity in a selected spot as compared to the average marker's activity in the whole body.

$$
\frac{\mathrm{SUV}=\text { marker's activity in the tissue }[\mathrm{Mbq} / \mathrm{ml}]}{\text { injected marker's activity }[\mathrm{Mbq} / \text { body mass }(\mathrm{g})]}
$$

SUV, expressed through a numeric value, makes it possible to interpret various areas of metabolic activity within a single test, and makes it easier to make comparisons between other PET tests which use different markers.

In our research we used maximal SUV (SUV max) evaluated through volumetric technique.

The SUV value for malignant cancer in PET/CT tests with fluorodeoxyglucose (FDG) currently in use usually exceeds 2 [10].

In our research we focused on the SUV gradient when fluorothymidine is used for detection of various stomach cancer types within the primary tumor, as well as metastatic lymph nodes and remote metastases.

\section{Results}

The clinical tumor advancement degree based upon diagnostic tests performed:

- local advancement T+NOMO: 6 patients,

- regional advancement T+N+M0: 22 patients,

- general advancement T+NxM1: 22 patients.

The correct evaluation of primary tumor in the FLT-PET/CT test was possible in all 50 patients (100\%). $98 \%$ of the results were truly positive and $2 \%$ were truly negative. 
In 1 patient, in whose case the test was carried out 8 months after inductive chemotherapy began, the test did not show presence of cancerous growth, neither the primary tumor nor lymph node nor remote metastases [FLT-PET/CT result $\mathrm{T}(-) \mathrm{N}(-) \mathrm{M}(-)]$.

In the group under scrutiny, the following histopathological primary cancer types were recognized:

- mucocellular/mucinous - 14 (of which 4 were in G3 degree of differentiation),

- tubular - 11 (of which 3 were in G3 degree of differentiation),

- adenocarcinoma of undefined type - 18 (of which 3 were in $\mathrm{G} 3$ degree of differentiation),

- tubular/partly mucinous - 1 ,

- low differentiated (without any definite type) - 6 .

The degree of cancer differentiation determined in diagnostic tissue samplings obtained from the primary tumor through endoscopy (this degree was determined only in 25 patients):

- G1 - determined in no patient,

- G2 - determined in 12 patients,

- G3 - determined in 13 patients,

- in 25 cases in the histopathology test of the samplings obtained through endoscopy from the primary tumor the pathomorphologist did not determine the cancer advancement degree at all.

In the test group of 50 patients, the metabolic increase of thymidine in regional lymph nodes indicating presence of metastatic lymph nodes was evident in 42 patients [N9(+) qualified] (84\% of patients under scrutiny).

In the test group of 50 patients, metastatic non-regional lymph nodes (e.g. para-aortic lymph nodes, nodes of the upper mesenteric artery area or nodes localized below the renal vessels) were described in 21 patients, which was $42 \%$ of the whole group.

In none of the patients did there occur a situation in which the test could not have detected metastases in lymph nodes of the perigastric area but could have detected remote metastases.

Remote nodal metastases were detected (in FLT-PET/CT scans) altogether in 5 patients (10\%):

- adrenal glands - 1 ,

- bones - 1 ,

- lung - 1 ,

- liver - 2 .

In none of the patients was any independent cancer other than stomach cancer detected.

In the FLT-PET/CT tests performed, the following values of SUV were obtained:

1) in primary tumor localization, in 49 patients FLT-PET/CT

$\mathrm{T}(+)$ uptake of FLT (SUVmax) was $7.59 \pm 3.86$ (2.9-17.7 range)

The value of SUVmax in primary tumor depending on histopathological tumor type was as follows:

- in mucinous/mucocellular cancer type (14 patients) 5.06 \pm 2.15 (2.8-10.2 range),

- in tubular cancer type (11 patients) $6.88 \pm 2.77$ (3.9-13.0 range),

- in cancer of undetermined type (18 patients) $9.33 \pm 4.19$ (2.817.7 range).
2) SUV $\max$ for regional lymph nodes:

a) results for 43 patients PET/CT N(+):

- FLT uptake $\left(S U V_{\max }\right)$ in regional lymph glands qualified as metastatic [in 43 patients PET/CT N(+)] equaled on average $3.93 \pm 2.88$ (1.3-14.1 range),

- FLT uptake (SUV max $_{\text {a }}$ in regional lymph nodes qualified as metastatic for mucinous/mucocellular cancer type equaled on average $3.54 \pm 1.58$ (1.5-7.7 range) (for 14 patients),

- FLT uptake (SUV $\left.V_{\text {max }}\right)$ in regional lymph nodes qualified as metastatic for tubular cancer type on average equaled $2.97 \pm 1.43$ (1.5-5.8 range) (for 9 patients),

- FLT uptake (SUV $\left.V_{\max }\right)$ in regional lymph glands qualified as metastatic in the rest of cases (qualified as indefinable cancer type) averaged $5.49 \pm 3.84$ (range 1.8-14.1) (in 19 patients);

b) results for a group of 14 patients, qualified for resection surgery: $\mathrm{hpN}(+)$ group:

- FLT uptake (SUV max $)$ in regional lymph nodes qualified as metastatic for mucinous/mucocellular cancer types (4 patients) - the average SUV $V_{\max }-3.5 \pm 1.09$ (2.5-4.0 range),

- FLT uptake (SUV $\left.V_{\max }\right)$ in regional lymph nodes described as metastatic for tubular cancer type (6 patients) - average SUVmax - $2.55 \pm 1.18$ (1.5-4.8 range),

- FLT uptake $\left(S U V_{\text {max }}\right)$ in regional lymph nodes described as metastatic in the remaining 4 cases (with non-qualified cancer type) - the average $S U V_{\max }-3.47 \pm 2.11$ (1.88.3 range);

3) for metastatic changes:

- in 21 patients PET/CT M(+) uptake of FLT $\left(S_{\text {max }}\right)$ in changes qualified as metastatic changes equaled 5.64 \pm 3.12 (1.5-11.9 range),

- FLT uptake (SUV $\max$ ) in changes qualified as metastatic in 7 patients with mucinous/mucocellular cancer type averaged $4.66 \pm 3.66$ (1.5-11.8 range),

- FLT uptake (SUV max ) in changes described as metastatic in 7 patients with tubular cancer type averaged 6.5 \pm 2.57 (1.7-8.4 range).

In 14 node positive patients the number of identified lymph nodes $\mathrm{N}+/ \mathrm{N}-(121 / 394)$ gives the average number of metastatic lymph nodes in one single test as 9 .

Detailed analysis was conducted on a group of 21 patients who had undergone resection.

The study involved assessment of $\mathrm{T}$ feature and $\mathrm{N}$ feature concordance in FLT-PET/CT examination and in histopathology:

1) T feature concordance:

- presence of primary tumor in FLT-PET/CT was identified in 20 cases,

- in 19 cases the test was true positive [FLT-PET/CT(+), hp$\mathrm{T}(-)]$,

- FLT-PET/CT was false negative in one patient, ${ }^{*}$ who had undergone two operations due to pyloric ulcer. The first surgery - distal gastric resection by Rydygier's method - was performed in 1984. The second operation - peripheral gastric resection with gastro-gastric anastomosis (by Billroth II method) performed due to stenosis and suspected recurrence of gastric ulcer at the site of anas- 
tomosis - was conducted in November 2010, two months before PET examination and surgery at the Oncology Center in Bydgoszcz (January 2011). The following was diagnosed in the tissue section excised during the second surgery (performed due to stenosis of gastro-gastric anastomosis): ca. mucocellulare ventriculi, Lauren II, gastritis chronica diffusa pT3Nx, "neoplastic infiltration involves the entire thickness of gastric wall, incision lines are free of neoplastic changes",

- there were no lymph nodes in the tissue section and diagnosis of cancer in this patient was based on the postoperative histopathological examination,

- despite finding no neoplastic changes at the margins of the section, the fact that width of tumor-free margins was not assessed and lack of lymph nodes in the specimen were considered as evidence of incomplete resection, prompting a decision to excise the remaining fragment of the stomach,

- total resection of the remaining part of the stomach was performed in January 2011 and the following result of histopathological examination was obtained: "omentum is free of neoplastic lesions, gastric stump (8x14 cm in dimensions) with intestinal anastomosis, an erosion $(0.4 \mathrm{~cm}$ in diameter) is present in the middle part of the stomach; mucosal membrane in this part of the stomach contains abundant inflammatory infiltrates; no neoplastic weaving was noted in the resected fragment of the stomach, in the area of anastomosis and intestine; no lymph nodes were found in the examined specimen",

- in this case a false positive result of FLT-PET/CT was most likely due to a small time interval between previous surgery and FLT-PET/CT examination. In such a short time inflammatory and regenerative processes that accompany healing of tissues after extensive surgery had possibly not completely subsided, which was reflected by increased fluorothymidine metabolism,

- one examination was true negative [FLT-PET/CT(-), hp-T(-)]; FLT-PET/CT was performed after a neoadjuvant chemotherapy and postoperative histopathological examination did not reveal presence of a primary tumor (the patient remains under observation).

2) N feature concordance:

- mean number of lymph nodes assessed during one histopathological examination was 25 ,

- mean number of lymph nodes containing metastatic lesions per one histopathological examination in patients pN(+) was 10.0 (range: 2/41-33/37).

a) pNO feature:

- in 4 cases where no nodal metastases were found in postoperative histopathological examination (hpNO) the result of FLT-PET/CT was also N(-), which constitutes $67 \%$ of true negative results,

- in 2 cases where no nodal metastases were found in postoperative histopathological examination (hpNo) the result of FLT-PET/CT was N(+), which constitutes 33\% of false positive results (lack of concordance between $\mathrm{N}(-)$ histopathology and N(+) FLT-PET/CT examination:
- the first case of hpNO and FLT-PET/CT(+) involved a patient previously subjected to gastric resection by Rydygier and BII methods - in this subject no lymph nodes were found in the tissue section obtained during present surgery ( ${ }^{*}$ this case is discussed above in detail together with a discussion on $T$ feature concordance), - in the second case of hpNO and FL-PET/CT(+) increased FLT uptake was noted in a single lymph node located outside the pylorus,

- in both of the above cases there is a possibility that lymph nodes exhibiting increased FLT metabolism visualized in PET/CT were not excised during surgery due to their localization at the margin of the area of resection: FLT$\mathrm{PET} / \mathrm{CT}$ was false positive in one patient;

b) $\mathrm{pN}(+)$ feature:

- nodal metastases were confirmed by histopathological examination in 14 patients; additionally, a single micrometastasis was found in 1 patient,

- in a group of 15 patients FLT-PET/CT examination confirmed the presence of nodal metastases in 11 cases, which constituted $73 \%$ of true positive results,

- in 4 cases FLT-PET/CT examination did not reveal presence of nodal metastases [FLT-PET/CT N(-) result] in $h p N(+)$ patients, which constituted $27 \%$ in this group (false negative results),

- FLT-PET/CT examination was N(-) in one patient with single node micrometastasis found in postoperative histopathological examination.

We assessed the degree of identification of primary tumor in FLT-PET/CT depending on the tumor size in postoperative histopathological examination (20 cases of resection):

- all tumors were FLT-PET T(+) regardless of size,

- mean maximal tumor size was 57 mm (range: 10-120 mm).

\section{Treatment}

Out of a group of 50 subjects 37 patients underwent surgery.

Twenty-one gastric resections were performed - 19 radical procedures and 2 palliative resections.

Sixteen non-resection procedures were performed (14 laparotomies, including 4 bypass procedures, 1 feeding jejunostomy and 1 diagnostic resection of liver metastasis).

Four patients were referred for induction chemotherapy:

- 2 patients on the basis of FLT-PET/CT examination,

- 2 patients after exploratory laparotomy.

Thirteen patients were not referred for surgical treatment. From this group, 10 patients were referred for palliative chemotherapy on the basis of FLT-PET/CT examination and 3 patients were referred for brachytherapy (one patient was referred for preoperative brachytherapy).

\section{Discussion}

Proper staging of the neoplastic process plays a key role in determining the subsequent therapeutic management. Preliminary determination of local tumor stage constitutes the basis for referral to surgical treatment. Surgical treatment or induction chemotherapy followed by surgery is possible in locally and regionally advanced tumors. However, identification of distant metastases shifts patients to the pallia- 
tive (surgical or chemotherapeutic) treatment group [3, 5]. After determining histopathological diagnosis on the basis of tissue sections collected during gastroscopy, imaging studies constitute the key element of diagnostics, allowing for assessment of tumor staging. The most common imaging studies used in the diagnostics of gastric cancer are the following: chest X-ray (assessment of metastatic lesions in lungs), ultrasound scan of the abdomen (assessment of metastases in the liver or other solid abdominal organs, assessment of metastases to periaortic lymph nodes) and computed tomography of the chest and abdomen, which carries the greatest amount of data (allowing for assessment of primary tumor, metastatic lesions in the enlarged lymph nodes, distant metastases in the liver, lungs and solid organs). Visualization of tumorous lesions or identification of enlarged lymph nodes is the basis for the diagnosis of a neoplastic process in those studies. Inability to evaluate the metabolism of visualized tissues as well as difficulty in assessment of small tumorous lesions and unenlarged (in radiological studies) lymph nodes constitute significant limitations of those diagnostic modalities [7].

Gastric cancer spreads through the lymphatic system hematogenously or through direct metastasis into the peritoneal cavity. Hematogenous or peritoneal spreading leads to formation of distal metastases and their identification determines palliative treatment. Identification of locoregional metastases to the surrounding lymph nodes or direct neoplastic infiltration facilitates radical treatment [5]. However, in cases of a high degree of local tumor advancement - extensive primary tumor and/or numerous lymph node metastases in locations determining the borders of regional spread (e.g. peripyloric or celiac trunk lymph nodes - respective groups 1,2 and 9 according to the Japanese Classification of Gastric Carcinoma) [21] - primary surgical intervention may not be the most beneficial management. In such cases resection is associated with great technical difficulty and the procedure itself is burdened with high risk of intraoperative neoplastic spread. In such instances it appears reasonable to implement induction chemotherapy aimed at reducing tumor volume and destroying micrometastatic lesions [3, 22, 23]. However, such management is not recommended in cases of metastases located outside the locoregional area. Thus, it is important to visualize all metastatic changes, particularly those located proximally to the primary tumor, but outside the locoregional area - e.g. supradiaphragmatic lymph nodes, retropancreatic lymph nodes, nodes in the area of the superior mesenteric artery or renal arteries. Such lesions may be identified in ultrasound or computed tomography examinations as long as they lead to lymph node enlargement. However, if lymph nodes remain unenlarged then confirmation of neoplastic involvement in this area is practically impossible. Positron emission tomography visualizes tissue metabolism and, in combination with simultaneous computed tomography, allows for precise localization of neoplastic lesions. PET-CT examination allows for exact determination of the extent of the neoplastic process.

${ }^{18} \mathrm{~F}$-fluorodeoxyglucose is a radiotracer widely used in tumor diagnostics. However, attempts at using FDG in PET/CT in the diagnostics of gastric cancer did not bring satisfactory results [13-19]. Due to low FDG metabolism in some histological types of gastric cancer (ca. mucocellulare, ca. mucinosum) the rate of identification of the primary lesion did not exceed $65 \%$. Therefore, studies on the use of other radiotracers in diagnostics of gastric cancer were undertaken. ${ }^{18} \mathrm{~F}$-fluorothymidine is one of those radiotracers. It is a substrate for thymidine kinase (an enzyme involved in thymidine monophosphate formation) and accumulates in rapidly proliferating cells present in malignant tumors.

Hermann et al. conducted a pilot study comparing FLTPET to FLT-PET/CT in the diagnostics of advanced gastric cancer [20]. The study included 45 patients. Neoplasms of intestinal type were diagnosed in 15 patients (33\%) and diffuse-type neoplasms were identified in the remaining 30 patients (67\%). $78 \%$ of studied tumors were characterized by a low grade of differentiation. Sensitivity of FLT-PET in identification of primary gastric tumor was 100\% (as many as $60 \%$ of tumors in the studied group contained signet ring cells and $40 \%$ possessed features of mucinous carcinoma). Pictures of proliferation in this study demonstrated a high degree of contrast for both primary tumors as well as for bone marrow proliferation. In 45/45 (100\%) patients with focal FLT uptake in the location of primary tumor the presence of a primary focus was confirmed through routine diagnostic modalities. As opposed to the results from FLT studies, only 31/45 (69\%) tumors were identified using FDG. Mean FLT uptake (SUV) in primary tumor locations amounted to $5.7 \pm 2.9$ (range: 2.0-12.9) and mean FDG uptake amounted to $6.7 \pm 4.6$ (range: 1.7-19.0; $p=0.09$ ). Comparison of mean FLT and FDG uptake in patients with low FDG uptake (SUV < 5) demonstrated statistically significant, higher FLT uptake (SUV $4.1 \pm 1.3$ vs. 3.0 $\pm 0.8 ; p=0.01$ ). In this first study comparing the assessment of locally advanced gastric cancer using FLT-PET and FLTPET/CT (low-dose CT), the FLT-PET method exhibited higher sensitivity than FDG-PET/CT (45/45 vs. 31/45 of identified gastric cancers). In the subgroup of patients with low FDG uptake, mean FLT uptake was significantly higher than mean FDG uptake. On the basis of high sensitivity of FLTPET in diagnosis of advanced gastric cancer, researchers proposed that there is a possibility of more precise staging of gastric cancer, particularly for histological subtypes with low FDG uptake (ca. mucocellulare ventriculi) [20].

There is a small number of international publications available on the discussed topic $[20,24]$. Our study is groundbreaking in that respect. Our evaluation of diagnostic efficacy of fluorothymidine in PET/CT in identification of neoplastic process is the first publication presenting the results of studies conducted in a Polish center. These results correspond to those obtained by Hermann and Kameyama and confirm high sensitivity with regard to identification of all histopathological types of gastric cancer. They allow patients to be referred for appropriate oncological management. On the basis of FLT-PET/CT patients with advanced locoregional $(66 \%)$ tumor stage were referred for induction chemotherapy, while patients with disseminated neoplasm (33\%) were offered palliative therapy. Further studies are required in order to formulate final conclusions on the role of PET/CT with ${ }^{18} \mathrm{~F}$-fluorothymidine. 
In conclusion:

1. FLT-PET/CT may be useful in the diagnostics and treatment of advanced gastric cancer.

2. Preliminary results of studies indicate that FLT-PET/CT examination is an effective method of assessment of primary tumor and regional lymph nodes.

3. Further studies and a greater number of subjects undergoing resection surgery are necessary, allowing for unequivocal determination of the value of FLT-PET/CT examination for the diagnostics and therapy of gastric cancer.

4. FLT-PET/CT examination facilitates making proper therapeutic decisions - it allows the number of unnecessary laparotomies to be lowered, while giving a chance to commence palliative chemotherapy in patients with metastatic disease or begin early induction treatment in patients with substantial locoregional tumor advancement.

The authors declare no conflict of interest.

\section{References}

1. Schmidt J, Gruca Z, Krawczyk M, et al. Podstawy chirurgii. Medycy na Praktyczna 2003; 691-714

2. Krzakowski M, Herman K, Jassem J et al. Zalecenia postępowania diagnostyczno- terapeutycznego w nowotworach złośliwych. Polska Unia Onkologii, Via Medica 2007; 108-119

3. Yoshikawa T, Sasako M, Yamamoto S, et al. Phase II study of neoad juvant chemotherapy and extended surgery for locally advanced gastric cancer. Br J Surg 2009; 96: 1015-22.

4. Kitaoka H, Yoshikawa K, Hirota T, Itabashi M. Surgical treatment of early gastric cancer. Jpn J Clin Oncol 1984; 14: 283-93.

5. Jeziorski A, Szawlowski A, Towpik E. Chirurgia onkologiczna. Wydawnictwo Lekarskie PZWL, Warszawa 2009; 1034-71.

6. Songun I, Putter H, Kranenbarg EM, Sasako M, van de Velde CJ. Surgical treatment of gastric cancer: 15 -year follow-up results of the randomised nationwide Dutch D1D2 trial. Lancet Oncol 2010; 11: 439-49.

7. Lim JS, Yun MJ, Kim MJ, Hyung WJ, Park MS, Choi JY, Kim TS, Lee JD, Noh SH, Kim KW. CT and PET in stomach cancer: preoperative staging and monitoring of response to therapy. Radiographics 2006; 26 143-56

8. Rohren EM, Turkington TG, Coleman RE. Clinical applications of PET in oncology. Radiology 2004; 231: 305-32.

9. Vallabhajosula S. (18)F-labeled positron emission tomographic radiopharmaceuticals in oncology: an overview of radiochemistry and mechanisms of tumor localization. Semin Nucl Med 2007; 37: 400-19.

10. Papathanassiou D, Bruna-Muraille C, Liehn JC, Nguyen TD, Curé H. Positron Emission Tomography in oncology: present and future of PET and PET/CT. Crit Rev Oncol Hematol 2009; 72: 239-54.

11. Kawamura T, Kusakabe T, Sugino T, Watanabe K, Fukuda T, Nashimoto A, Honma K, Suzuki T. Expression of glucose transporter-1 in human gastric carcinoma. Cancer 2001; 92: 634-41.

12. Staniuk T, Jankowski M, Zegarski W, Małkowski B, Kula Z. Evaluation of the usefulness of FDG-PET/CT in diagnosis and qualification for surgical treatment of advanced gastric cancer. Wspolczesna Onkol 2010; 14: 302-9.

13. Yun M, Lim JS, Noh SH, Hyung WJ, Cheong JH, Bong JK, Cho A, Lee JD. Lymph node staging of gastric cancer using (18) F-FDG PET: a com parison study with CT. J Nucl Med 2005; 46: 1582-8.

14. Chen J, Cheong JH, Yun MJ, Kim J, Lim JS, Hyung WJ, Noh SH. Improvement in preoperative staging of gastric adenocarcinoma with positron emission tomography. Cancer 2005; 103: 2383-90.

15. Stahl A, Ott K, Weber WA, Becker K, Link T, Siewert JR, Schwaiger M, Fink U. FDG PET imaging of locally advanced gastric carcinomas: correlation with endoscopic and histopathological findings. Eur J Nucl Med Mol Imaging 2003; 30: 288-95.
16. Mukai K, Ishida Y, Okajima K, Isozaki H, Morimoto T, Nishiyama S. Usefulness of preoperative FDG-PET for detection of gastric cancer. Gastric Cancer 2006; 9: 192-6.

17. Mochiki E, Kuwano H, Katoh H, Asao T, Oriuchi N, Endo K. Evaluation of 18F-2-deoxy-2-fluoro-D-glucose positron emission tomography for gastric cancer. World J Surg 2004; 28: 247-53.

18. Yeung HW, Macapinlac H, Karpeh M, Finn RD, Larson SM. Accuracy of FDG-PET in Gastric Cancer. Preliminary Experience. Clin Positron Imaging 1998; 1: 213-21.

19. Yoshioka T, Yamaguchi K, Kubota K, et al. Evaluation of 18F-FDG PET in patients with a, metastatic, or recurrent gastric cancer, J Nucl Med 2003; 44: 690-9.

20. Herrmann K, Ott K, Buck AK, Lordick F, et al. Imaging gastric cancer with PET and the radiotracers 18F-FLT and 18F-FDG: a comparative analysis. J Nucl Med 2007; 48: 1945-50.

21. Japanese Gastric Cancer Association. Japanese classification of gastric carcinoma: 3rd English edition. Gastric Cancer 2011; 14:101-12.

22. Eguchi T, Kodera Y, Nakanishi H, et al. The effect of chemotherapy against micrometastases and isolated tumor cells in lymph nodes: an in vivo study. In Vivo 2008; 22: 707-12.

23. Popiela T, Kulig J, Czupryna A,et al. Eradication of micrometastases in bone marrow and cancer cells in peripheral blood using preoperative chemotherapy. ASCO 2004. Gastrointestinal Cancers Symposium, Session Type and Session Titler: Cancers of the Esophagus and Stomach- General poster session. Abstract No. 12.

24. Kameyama R, Yamamoto Y, Izuishi K, Takebayashi R, Hagiike M, Murota M, Kaji M, Haba R, Nishiyama Y. Detection of gastric cancer using 18F-FLT PET: comparison with 18F-FDG PET. Eur J Nucl Med Mol Imaging 2009; 36: 382-8.

\section{Address for correspondence}

Tomasz Staniuk

Oncology Centre - Prof. Lukaszczyk Memorial Hospital

dr I. Romanowskiej 2

85-796 Bydgoszcz, Poland

e-mail: tomasz_staniuk@tlen.pl

Submitted: 12.06 .2012

Accepted: 07.08.2012 